\title{
Generalized Sequences with Applications to the Discrete Calculus
}

\author{
By J. F. Traub
}

1. Introduction. Mikusiński [17] has introduced a theory of generalized functions which is algebraic in nature. Generalized functions are introduced in a way which is analogous to the extension of the concept of number from integers to rationals.

In this paper, an analogous theory of "generalized sequences" is constructed for the discrete calculus. This theory serves a dual purpose. It provides a rigorous foundation for an operational calculus and provides a powerful formalism for the solution of discrete problems.

The algebraic formalism of generalized sequences is given in Sections 2 and 3, while the relations between such operators as $E, \Delta, M, \Delta^{-1}, M^{-1}$ and certain generalized sequences are discussed in Sections 4 and 8. Newton's interpolation formula with a summation remainder, which is analogous to Taylor's formula with an integral remainder, is obtained in Section 6. A general solution of the $n$ th-order inhomogeneous difference equation with constant coefficients is given in Section 7 , in connection with which the reader is referred to the discussion of partial fraction expansions in Appendix A. The discrete analogues of Heaviside's unit step function and Dirac's delta function occur in a natural way and are discussed in Sections 9 and 10.

The relation between generalized sequences and such "classical" operational methods as generating functions, Dirichlet transforms, and $z$-transforms is discussed in Section 12. Automation of the solution of various types of linear problems is discussed in Appendix B.

A few words on the use of the term "discrete calculus" are in order. A more complete title might be "real functions of a discrete variable." This subject is usually referred to as the "calculus of finite differences." However, the basic operator of this calculus is the translation operator and not the difference operator. Moreover, the word finite has various meanings. It sometimes means noninfinite, sometimes nonzero, and sometimes noninfinitesimal. In the present context it refers to noninfinitesimal and is a relic from the days of the "infinitesimal calculus."

Hence we refer to the subject as the discrete (as opposed to continuous) calculus. We restrict ourselves to the case where the discrete variable takes on equally spaced values on a half-line. It is sufficient to consider the case where these values are the nonnegative integers and we therefore deal with sequences.

2. The Field of Generalized Sequences. Let $S$ be a set of sequences. Let $\{f\} \in S$. Then

$$
\{f\}=f(0), f(1), f(2), \cdots .
$$

(This notation is used instead of $f_{0}, f_{1}, f_{2}$ to emphasize that we are dealing with a

Received August 17, 1964. Presented to the 69th Summer Meeting of the American Mathematical Society, Amherst, Massachusetts, August 1964. 
function of a discrete variable and to avoid the use of subscripts.) When there is no danger of confusion, we write $\{f\}$ as $f$. The notation $f(\lambda)$ is also used.

Two sequences are equal if

$$
f(\lambda)=g(\lambda), \quad \lambda=0,1,2, \cdots .
$$

Addition of sequences is componentwise. Thus

$$
h=f+g
$$

if

$$
h(\lambda)=f(\lambda)+g(\lambda), \quad \lambda=0,1 ; 2, \cdots .
$$

Multiplication of two sequences is defined as their convolution. Thus

$$
h=f g
$$

if

$$
h(\lambda)=\sum_{j=0}^{\lambda} f(j) g(\lambda-j) .
$$

Concatenation of two sequences denotes their multiplication as defined above. To indicate componentwise multiplication we use a dot. Thus

$$
h=f \cdot g
$$

if

$$
h(\lambda)=f(\lambda) g(\lambda), \quad \lambda=0,1,2, \cdots .
$$

Our use of componentwise multiplication will be rare.

It is easy to verify that if $f, g, h \in S$, then

1. $f+g=g+f$.

2. $f+(g+h)=(f+g)+h$.

3. $f+x=g$ has a unique solution $x \in S$.

4. $f g=g f$.

5. $f(g h)=(f g) h$.

6. $f(g+h)=f g+f h$.

Hence we have a commutative ring. The additive identity is $0,0,0, \cdots$. The multiplicative identity is $1,0,0, \cdots$. We now show that our commutative ring is an integral domain by verifying that if $f g=\{0\}, f \neq\{0\}$, then $g=\{0\}$. Thus, by hypothesis,

$$
\sum_{j=0}^{\lambda} f(j) g(\lambda-j)=\{0\} .
$$

Assume $f(0) \neq 0$. Then by successively setting $\lambda=0,1,2, \cdots$, we calculate $g(0), g(1), g(2), \cdots$, to be zero.

Now assume $f(0)=0$. Since $f$ is not the zero sequence, there is a first element $f(m) \neq 0$. Therefore

$$
\sum_{j=m}^{\lambda} f(j) g(\lambda-j)=\{0\} .
$$


Then by successively setting $\lambda=m, m+1, m+2, \cdots$, we calculate $g(0)$, $g(1), g(2), \cdots$, to be zero. Hence the result is proved.

In the continuous case, the analogous theorem, due to Titchmarsh, is highly nontrivial.

Our ring is an integral domain and may therefore be embedded in a field. This embedding is analogous to the embedding of the integral domain of integers in the field of rational numbers.

For given $f$ and $g$, there may or may not be a sequence $x$ such that $g=f x$. We define the fraction $g / f, f \neq\{0\}$ as the solution of $g=f x$. The symbol $g / f$ denotes the inverse to convolution. The solution is uniquely determined. For, let $g=f x_{1}, g=f x_{2}, f \neq\{0\}$. Then $\{0\}=f\left(x_{1}-x_{2}\right)$ and $x_{1}-x_{2}=\{0\}$.

We call the equivalence class of fractions $g / f$ generalized sequences. We shall sometimes call them operators. Every sequence $f$ may be written as a generalized sequence $f g / g$, but not every generalized sequence is a sequence. A subset of the field of generalized sequences is isomorphic to the ring of sequences. The scope of generalized sequences is treated in Section 11.

Let $f, h \neq\{0\}$. Equality, addition, and multiplication are defined by

$$
\begin{aligned}
\frac{e}{f} & =\frac{g}{h} \quad \text { if and only if } e h=f g, \\
\frac{e}{f} \frac{g}{h} & =\frac{e g}{f h}, \\
\frac{e}{f}+\frac{g}{h} & =\frac{e h+f g}{f h} .
\end{aligned}
$$

Scalars are sequences of the form $[a]=a, 0,0, \cdots$. Observe that

$$
\begin{aligned}
{[a]+[b] } & =[a+b] . \\
{[a][b] } & =[a b] .
\end{aligned}
$$

Scalars should not be confused with constant sequences

$$
\{a\}=a, a, a, \cdots \text {. }
$$

For constant sequences, we have

$$
\{a\}\{b\}=a b, 2 a b, 3 a b, \cdots,
$$

rather than (2.1). Scalars are isomorphic to numbers. Let $\sigma$ denote the sequence

$$
\sigma=1,1,1, \cdots \text {. }
$$

If $\{a\}$ is a constant sequence, then the scalar $[a]$ may be written as

$$
[a]=\frac{\{a\}}{\sigma} .
$$

Two important scalars were introduced above. They are

$$
\begin{aligned}
& {[0]=0,0,0, \cdots,} \\
& {[1]=1,0,0, \cdots .}
\end{aligned}
$$


Observe that $[0] \equiv\{0\}$. Since scalars are isomorphic to numbers we write [0] and [1] as 0 and 1 .

3. The Sequence $\omega$. Let $\omega$ denote the sequence

$$
\omega=0,1,0,0, \cdots \text {. }
$$

The sequence $\omega^{j}$ (where the power denotes a $j$-fold convolution) is the sequence with a 1 in the $j$ th position and 0 elsewhere. We define $\omega^{0}=1$.

The sequence

$$
\sigma=1,1,1, \cdots .
$$

was introduced in Section 2. Another important sequence is

$$
\nu=1,-1,1,-1, \cdots \text {. }
$$

The relation of $\omega, \sigma, \nu$ to the operators $E^{-1}, \Delta^{-1}, M^{-1}$ of the discrete calculus is given in Sections 4 and 8 . Inverses to $\omega, \sigma, \nu$ are defined by

$$
\begin{aligned}
& \omega \tau=1, \\
& \sigma \rho=1, \\
& \nu \mu=1 .
\end{aligned}
$$

Direct calculation shows that $\rho$ and $\mu$ are sequences given by

$$
\begin{aligned}
& \rho=1,-1,0,0, \cdots, \\
& \mu=1,1,0,0, \cdots .
\end{aligned}
$$

On the other hand, $\tau$ is not a sequence. It is a generalized sequence. The basic sequences are summarized in Table 1.

Observe that

$$
\begin{aligned}
& \rho=1-\omega, \\
& \mu=1+\omega .
\end{aligned}
$$

Using (3.1) and (3.2), any of the six operators $\omega, \sigma, \nu, \tau, \rho$, or $\mu$ may be expressed in terms of any of the other five. We elect to express these operators in terms of $\omega$.

TABLE 1

The Basic Sequences

\begin{tabular}{c|l}
\hline Symbol & \multicolumn{1}{|c}{ Sequence } \\
\hline 0 & $0,0,0, \ldots$ \\
1 & $1,0,0, \ldots$ \\
$\omega$ & $0,1,0,0, \ldots$ \\
$\sigma$ & $1,1,1, \ldots$ \\
$\nu$ & $1,-1,1,-1, \ldots$ \\
$\rho$ & $1,-1,0,0, \ldots$ \\
$\mu$ & $1,1,0,0, \cdots$ \\
\hline
\end{tabular}


Thus

$$
\begin{aligned}
& \sigma=\frac{1}{1-\omega}, \\
& \nu=\frac{1}{1+\omega}, \\
& \tau=\frac{1}{\omega}, \\
& \rho=1-\omega, \\
& \mu=1+\omega .
\end{aligned}
$$

Observe that if $f$ is an arbitrary sequence (not a generalized sequence), then the sequence may be exhibited in terms of its members through

$$
f=\sum_{j=0}^{\infty} f(j) \omega^{j} .
$$

This important relation is used in Sections 11 and 12.

Direct calculation shows that the sequence $1,-a, 0,0, \cdots$ is the inverse of the sequence $1, a, a^{2}, \cdots$. The sequence $1,-a, 0,0, \cdots$ may be written as 1 $a \omega$ where $a$ is a scalar. Since $1, a, a^{2}, \cdots$ is the sequence $a^{\lambda}$, we have

$$
a^{\lambda}(1-a \omega)=1
$$

or

$$
a^{\lambda}=\frac{1}{1-a \omega}
$$

By convolution,

$$
\frac{1}{1-a \omega} \frac{1}{1-a \omega}=\frac{1}{(1-a \omega)^{2}}=\sum_{j=0}^{\lambda} a^{j} a^{\lambda-j}
$$

Therefore,

$$
\frac{1}{(1-a \omega)^{2}}=(\lambda+1) a^{\lambda}
$$

By induction,

$$
\frac{1}{(1-\boldsymbol{a} \omega)^{m+1}}=C(\lambda+m, m) a^{\lambda},
$$

where $C(\lambda, m)$ denotes the binomial coefficient

$$
C(\lambda, m)=\frac{1}{m !} \prod_{i=0}^{m-1}(\lambda-i) .
$$

Observe that if $f$ is an arbitrary sequence, then

$$
\begin{aligned}
\omega^{j} f(\lambda) & =f(\lambda-j), \quad \lambda \geqq j, \\
& =0, \quad \lambda<j .
\end{aligned}
$$


In particular,

$$
\frac{\omega^{m}}{(1-a \omega)^{m+1}}=C(\lambda, m) a^{\lambda-m}
$$

and

$$
\frac{\omega^{m}}{(1-\omega)^{m+1}}=C(\lambda, m)
$$

Since $C(\lambda, m)=0$ for $\lambda$ and $m$ nonnegative integers and $\lambda<m$, we need not write these relations for two cases as in (3.6).

Observe that in (3.7), $m$ is fixed and the sequence is indexed by $\lambda$, the first parameter of the binomial coefficient. Consider now the sequence

$$
1, a, 0,0, \cdots=1+a \omega \text {. }
$$

By convolution,

$$
(1+a \omega)^{2}=1+2 a \omega+a^{2} \omega^{2}=C(2, \lambda) a^{\lambda},
$$

and by induction,

$$
(1+a \omega)^{m}=C(m, \lambda) a^{\lambda} .
$$

Observe the similarity between (3.9) and the identity

$$
(1+a t)^{m}=\sum_{\lambda=0}^{m} C(m, \lambda) a^{\lambda} t^{\lambda} .
$$

We return to this in Section 12.

It is important not to confuse (3.7) and (3.8). For each $m$, the sequence

$$
\{C(\lambda, m)\}=C(0, m), C(1, m), C(2, m), \cdots
$$

has an infinite number of nonzero elements. On the other hand, only the first $m+1$ members of the sequence

$$
\{C(m, \lambda)\}=C(m, 0), C(m, 1), C(m, 2), \cdots
$$

are nonzero. For example,

$$
\begin{aligned}
& \{C(\lambda, 3)\}=\left\{\frac{\lambda(\lambda-1)(\lambda-2)}{6}\right\}=0,0,0,1,4,10, \cdots, \\
& \{C(3, \lambda)\}=1,3,3,1,0,0,0, \cdots .
\end{aligned}
$$

Formulas relating $\{C(\lambda, m)\}$ and $\{C(m, \lambda)\}$ are derived in Section 9.

We turn to the case where $a$ is complex. Let $a=R e^{i \varphi}$. Then

$$
\frac{\omega^{m}}{(1-a \omega)^{m+1}}=C(\lambda, m) a^{\lambda-m}
$$

becomes

$$
\frac{\omega^{m}}{\left(1-R e^{i \varphi} \omega\right)^{m+1}}=C(\lambda, m) R^{\lambda-m} e^{i(\lambda-m) \varphi} .
$$


The left side of (3.10) may be written as

$$
\frac{\omega^{m}}{\left(1-R e^{i \varphi} \omega\right)^{m+1}} \frac{\left(1-R e^{-i \varphi} \omega\right)^{m+1}}{\left(1-R e^{-i \varphi} \omega\right)^{m+1}}=\frac{\omega^{m} \sum_{k=0}^{m+1}(-1)^{k} C(m+1, k) R^{k} e^{-i k \varphi} \omega^{k}}{\left(1-2 R \omega \cos \varphi+R^{2} \omega^{2}\right)^{m+1}} .
$$

Taking real and imaginary parts of (3.10) and (3.11) leads to

$$
\begin{aligned}
& C(\lambda, m) R^{\lambda-m} \cos (\lambda-m)_{\varphi}=\frac{\omega^{m} \sum_{k=0}^{m+1}(-1)^{k} C(m+1, k) R^{k} \omega^{k} \cos k \varphi}{\left(1-2 R \omega \cos \varphi+R^{2} \omega^{2}\right)^{m+1}}, \\
& C(\lambda, m) R^{\lambda-m} \sin (\lambda-m)_{\varphi}=\frac{\omega^{m} \sum_{k=0}^{m+1}(-1)^{k-1} C(m+1, k) R^{k} \omega^{k} \sin k \varphi}{\left(1-2 R \omega \cos \varphi+R^{2} \omega^{2}\right)^{m+1}} .
\end{aligned}
$$

In particular,

$$
\begin{aligned}
R^{\lambda} \cos \lambda \varphi & =\frac{1-R \omega \cos \varphi}{1-2 R \omega \cos \varphi+R^{2} \omega^{2}} \\
R^{\lambda} \sin \lambda \varphi & =\frac{R \omega \sin \varphi}{1-2 R \omega \cos \varphi+R^{2} \omega^{2}} \\
R^{\lambda} \cos \frac{\pi}{2} \lambda & =\frac{1}{1+R^{2} \omega^{2}} \\
R^{\lambda} \cos \frac{\pi}{2} \lambda & =\frac{R \omega}{1+R^{2} \omega^{2}} .
\end{aligned}
$$

From (3.14) and (3.15), we may conclude that

$$
\frac{R^{\lambda} \sin \varphi(\lambda+1)}{\sin \varphi}=\frac{1}{1-2 R \omega \cos \varphi+R^{2} \omega^{2}},
$$

and from (3.6),

$$
\frac{R^{\lambda-1} \sin \varphi \lambda}{\sin \varphi}=\frac{\omega}{1-2 R \omega \cos \varphi+R^{2} \omega^{2}} .
$$

Using (3.1) and (3.2), these formulas may be written in terms of $\sigma$, $\tau$, etc. Thus $\omega=1 / \tau$ and (3.17) imply

$$
R^{\lambda} \sin \frac{\pi}{2} \lambda=\frac{R \tau}{R^{2}+\tau^{2}}
$$

We restrict ourselves to $m$ a nonnegative integer in formulas such as

$$
\frac{\omega^{m}}{(1-a \omega)^{m+1}}=C(\lambda, m) a^{\lambda-m}
$$

This formula could be extended to noninteger $m$ but we shall not do so here.

4. The Operators of the Discrete Calculus: $E, \Delta, M$. The standard operators of the discrete calculus have certain desirable algebraic properties. They lack certain other properties such as the possession of inverses. (The so-called inverse operators 
are not true inverses.) On the other hand, the operators introduced in Section 3 are members of a field. As we shall show in this section, there are simple relations between the two sets of operators. Hence to solve a problem in the discrete calculus, we translate into a problem involving generalized sequences, solve, and then translate back.

Three standard operators of the discrete calculus are defined by

$$
\begin{aligned}
E f(\lambda) & =f(\lambda+1), \\
\Delta f(\lambda) & =f(\lambda+1)-f(\lambda), \\
M f(\lambda) & =\frac{1}{2}[f(\lambda+1)+f(\lambda)] .
\end{aligned}
$$

$E, \Delta$, and $M$ are called the translation, forward differencè, and mean operator, respectively. The operators $\Delta^{-1}$ and $M^{-1}$ are introduced in Section 8 . Observe that $E f$ is not a convolution. The operator $E$ has no meaning in itself, but only in its effect on $f$. It is easy to verify that there exists no sequence $\kappa$, independent of $f$, such that

$$
\kappa f(\lambda)=f(\lambda+1)-f(\lambda)
$$

where $\alpha f$ is a convolution.

Multiply

$$
E f(\lambda)=f(\lambda+1)
$$

by $\omega$ and recall that

$$
\begin{aligned}
\omega f(\lambda) & =f(\lambda-1), & & \lambda \geqq 1, \\
& =0, & & \lambda=0 .
\end{aligned}
$$

Hence

$$
\omega E f=f-f(0) .
$$

(To understand sequence relations such as (4.2), the reader should recall that the scalar $f(0)$ is the sequence $f(0), 0,0, \cdots$.) Since $\tau \omega=1$, we conclude that

$$
E f=\tau f-\tau f(0),
$$

or

$$
E f=\frac{1}{\omega} f-\frac{1}{\omega} f(0) .
$$

Hence the generalized sequence $\tau$ is related to the translation operator. Also,

$$
\Delta f=(\tau-1) f-\tau f(0),
$$

or

$$
\Delta f=\rho \tau f-\tau f(0)
$$

where $\rho$ is the sequence

$$
1,-1,0,0, \cdots=1-\omega
$$


Hence the sequence $\rho$ is related to the forward difference operator. Furthermore

$$
M f=\frac{\mu}{2} \tau f-\frac{\tau}{2} f(0) .
$$

Hence $\mu / 2$ is related to the mean operator.

Equations (4.4) to (4.6) are of the form

$$
L f=\alpha f-\beta f(0),
$$

where $L$ is an operator of the discrete calculus and $\alpha$ and $\beta$ are generalized sequences. Replace $f$ by $L f$ in (4.7). Then

$$
\begin{aligned}
L^{2} f & =\alpha[\alpha f-\beta f(0)]-\beta L f(0) \\
& =\alpha^{2} f-\alpha \beta f(0)-\beta L f(0) .
\end{aligned}
$$

By induction,

$$
L^{n} f=\alpha^{n} f-\beta \sum_{j=0}^{n-1} \alpha^{n-1-j} L^{j} f(0) .
$$

In particular,

$$
\begin{aligned}
E^{n} f & =\tau^{n} f-\sum_{j=0}^{n-1} \tau^{n-j} f(j) \\
\Delta^{n} f & =\rho^{n} \tau^{n} f-\tau \sum_{j=0}^{n-1}(\rho \tau)^{n-1-j} \Delta^{j} f(0), \\
M^{n} f & =\left(\frac{\mu}{2}\right)^{n} \tau^{n} f-\frac{\tau}{2} \sum_{j=0}^{n-1}\left(\frac{\mu r}{2}\right)^{n-1-j} M^{j} f(0) .
\end{aligned}
$$

These relations show that the operators of the discrete calculus and certain of our generalized sequences differ only by terms involving "initial conditions." In Section 6, we demonstrate that (4.10) is essentially Newton's interpolation formula with remainder.

Although generalized sequences commute and many operators of the discrete calculus commute, the two families of operators do not commute with each other. Thus

$$
\left(\rho E^{\prime}-E_{\rho}^{\prime}\right) f=f(0) \text {. }
$$

5. The Summation Sequence $\sigma$. We defined

$$
\sigma=1,1,1, \cdots \text {. }
$$

Hence

$$
\sigma f=\sum_{j=0}^{\lambda} f(j)
$$

Since

$$
\sigma=\frac{1}{1-\omega}, \quad \sigma^{m}=\frac{1}{(1-\omega)^{m}} .
$$


From (3.5),

$$
\frac{1}{(1-\omega)^{m}}=C(\lambda+m-1, m-1) .
$$

Since $\sigma^{m} f$ may be interpreted as an $m$-fold sum or as the convolution of the sequence $\sigma^{m}$ with the sequence $f$, we conclude that

$$
\sum_{j_{m-1}=0}^{\lambda} \ldots \sum_{j_{1}=0}^{j_{2}} \sum_{j_{0}=0}^{j_{1}} f\left(j_{0}\right)=\sum_{k=0}^{\lambda} C(\lambda+m-1-k, m-1) f(k) .
$$

This is the analogue of Cauchy's formula

$$
\int_{0}^{t} d t_{m-1} \cdots \int_{0}^{t_{2}} d t_{1} \int_{0}^{t_{1}} f\left(t_{0}\right) d t_{0}=\int_{0}^{t} \frac{(t-u)^{m-1}}{(m-1) !} f(u) d u .
$$

Numerous expressions for sums of binomial coefficients may be derived by using algebraic identities in $\omega$. We give only a few examples here. See also Section 9. Thus,

$$
\frac{1}{1-\omega}(1-\omega)^{m}=(1-\omega)^{m-1}
$$

and (3.9) lead to the identity in $\lambda$,

$$
\sum_{j=0}^{\lambda}(-1)^{j} C(m, j)=(-1)^{\lambda} C(m-1, \lambda) .
$$

Also,

$$
(1+\omega)^{k}(1+\omega)^{m}=(1+\omega)^{k+m}
$$

and (3.9) lead to

$$
\sum_{j=0}^{\lambda} C(k, j) C(m, \lambda-j)=C(k+m, \lambda) .
$$

This is the well-known Vandermonde convolution. From

$$
\frac{\omega^{k}}{(1-\omega)^{k+1}} \frac{\omega^{m}}{(1-\omega)^{m+1}}=\frac{\omega^{k+m}}{(1-\omega)^{k+m+2}},
$$

we obtain

$$
\sum_{j=0}^{\lambda} C(j, k) C(\lambda-j, m)=C(\lambda+1, k+m+1) .
$$

If $m=\mathbf{0}$,

$$
\sum_{j=0}^{\lambda} C(j, k)=C(\lambda+1, k+1) .
$$

Convolution with $1 /(1-\omega)$ leads to summation. Convolution with $1 /(1+\omega)$ leads to summation of alternate sequences. Thus the identity

$$
\frac{1}{1+\omega} \frac{\omega}{(1-a \omega)^{2}}=-\frac{1}{(a+1)^{2}} \frac{1}{1+\omega}-\frac{a}{(a+1)^{2}} \frac{1}{1-a \omega}+\frac{1}{a+1} \frac{1}{(1-a \omega)^{2}}
$$


leads, after some simplification, to

$$
\sum_{j=0}^{\lambda}(-1)^{j} j a^{j-1}=\frac{1}{(a+1)^{2}}\left\{-1+(-a)^{\lambda}[1+\lambda(a+1)]\right\} .
$$

6. Newton's Interpolation Formula. Newton's interpolation formula is the discrete analogue of Taylor's formula. Taylor's formula with integral remainder is given by

$$
f(t)=\sum_{j=0}^{n} \frac{f^{(j)}(0)}{j !} t^{j}+\int_{0}^{t} \frac{(t-u)^{n}}{n !} f^{(n+1)}(u) d u .
$$

The derivative form of the remainder may be obtained from the integral form by the second law of the mean. On the other hand, Newton's interpolation formula is invariably given with a derivative remainder. We shall obtain the analogue of (6.1) for Newton's formula with a summation remainder replacing the integral remainder. From this we obtain the derivative form of the remainder.

We showed in Section 4 that

$$
\Delta^{n} f=\rho^{n} \tau^{n} f-\tau \sum_{j=0}^{n-1}(\rho \tau)^{n-1-j} \Delta^{j} f(0) .
$$

Solving for $f$, and using $\rho=1-\omega, \tau=1 / \omega$,

$$
f=\sum_{j=0}^{n} \frac{\omega^{j}}{(1-\omega)^{i+1}} \Delta^{j} f(0)+\left(\frac{\omega}{1-\omega}\right)^{n+1} \Delta^{n+1} f .
$$

This is Newton's formula with the remainder expressed as a convolution. Using (3.6) and (3.8), we can write (6.2) as

$$
f(\lambda)=P_{n}(\lambda)+R_{n}(\lambda),
$$

where

$$
\begin{aligned}
& P_{n}(\lambda)=\sum_{j=0}^{n} C(\lambda, j) \Delta^{j} f(0), \\
& R_{n}(\lambda)=\sum_{j=0}^{\lambda-1} C(\lambda-1-j, n) \Delta^{n+1} f(j) .
\end{aligned}
$$

Observe that the remainder differs from the integral form of the remainder of Taylor's formula in exactly the fashion that (5.1) differs from (5.2). This must be the case since Taylor's formula is an identity due to repeated integration by parts whereas Newton's formula is an identity due to repeated summation by parts. Observe that (6.3) is valid with no regularity assumptions.

If $\Delta^{n+1} f(j)$ is independent of $j$, then

and, using (5.3),

$$
R_{n}(\lambda)=\Delta^{n+1} f(0) \sum_{j=0}^{\lambda-1} C(\lambda-1-j, n),
$$

$$
R_{n}(\lambda)=\Delta^{n+1} f(0) C(\lambda, n+1) .
$$

Hence, for this case,

$$
f(\lambda)=\sum_{j=0}^{n+1} C(\lambda, j) \Delta^{j} f(0) .
$$


We return to the general case. Note that $R_{n}(\lambda)=0$ for $\lambda=0,1, \cdots, n$. Let

$$
a_{j}(\lambda, n)=\frac{C(\lambda-1-j, n)}{C(\lambda, n+1)}, \quad \lambda>n .
$$

Then

$$
\begin{aligned}
a_{j}(\lambda, n) & \geqq 0, \quad j=0,1, \cdots, \lambda-1 ; \\
\sum_{j=0}^{\lambda-1} a_{j}(\lambda, n) & =1 .
\end{aligned}
$$

We can write the remainder as

$$
R_{n}(\lambda)=C(\lambda, n+1) \sum_{j=0}^{\lambda-1} a_{j}(\lambda, n) \Delta^{n+1} f(j) .
$$

Thus the remainder is the product of a polynomial in $\lambda$ of degree $n+1$ with a weighted average of $(n+1)$ st differences.

This result has been obtained without a regularity hypothesis. It is an identity in $\lambda$ valid for nonnegative integer $\lambda$. We extend the result to arbitrary $\lambda$ and obtain the usual derivative form of the remainder as follows.

Let $f$ denote a function of a real variable and let $f^{(n+1)}$ exist. Then, for some $\xi_{j}$,

$$
\Delta^{n+1} f(j)=f^{(n+1)}\left(\xi_{j}\right) .
$$

Hence

$$
R_{n}(\lambda)=C(\lambda, n+1) \sum_{j=0}^{\lambda-1} a_{j}(\lambda, n) f^{(n+1)}\left(\xi_{j}\right) .
$$

The sum is a weighted average of $(n+1)$ st derivatives. By the intermediatevalue property of derivatives, there exists a point $\theta$ such that

$$
R_{n}(\lambda)=C(\lambda, n+1) f^{(n+1)}(\theta) .
$$

(Note that continuity of the $(n+1)$ st derivative is not required. Existence of the derivative implies the intermediate-value property.)

Hence

$$
f(\lambda)=\sum_{j=0}^{n} C(\lambda, j) \Delta^{j} f(0)+C(\lambda, n+1) f^{(n+1)}(\theta) .
$$

This formula is valid for arbitrary $\lambda$.

7. Solution of Difference Equations. The $n$ th-order linear difference equation with constant coefficients may be written as

$$
f(\lambda+n)+\alpha_{1} f(\lambda+n-1)+\cdots+\alpha_{n} f(\lambda)=g(\lambda),
$$

or

$$
\sum_{j=0}^{n} \alpha_{n-j} E^{j} f=g, \quad \alpha_{0}=1 .
$$

The coefficients can be real or complex. If $f(0), f(1), \cdots, f(n-1)$ are specified, the solution is uniquely determined for all $\lambda$. Equation (7.1) might better be called 
a recurrence relation; the term difference equation is used for historical reasons. The polynomial $\sum_{j=0}^{n} \alpha_{n-j} t^{j}$ is called the characteristic polynomial and the equation

$$
\sum_{j=0}^{n} \alpha_{n-j} t^{j}=0
$$

is called the characteristic or indicial equation. The roots of the equation

$$
\sum_{j=0}^{n} \alpha_{j} t^{j}=0
$$

are the reciprocals of the roots of (7.2). The sequence $g$ is sometimes called the forcing sequence.

We begin by considering the first-order equation

$$
\left(E+\alpha_{1}\right) f=g \text {. }
$$

From (4.4),

$$
E f=\frac{1}{\omega} f-\frac{1}{\omega} f(0)
$$

Hence

$$
\frac{1+\alpha_{1} \omega}{\omega} f=\frac{1}{\omega} f(0)+g .
$$

Therefore,

$$
f=\frac{f(0)}{1+\alpha_{1} \omega}+\frac{\omega}{1+\alpha_{1} \omega} g .
$$

From (3.5) and (3.6),

$$
f(\lambda)=f(0)\left(-\alpha_{1}\right)^{\lambda}+\sum_{j=0}^{\lambda-1}\left(-\alpha_{1}\right)^{\lambda-1-j} g(j) .
$$

This analysis exhibits a number of features which hold true in the $n$ th-order case. The particular solution is expressed as a convolution. As we shall see below, the form of $g$ may be such that we can evaluate this convolution without writing it as a sum. It is not necessary to obtain the general solution of the homogeneous equation plus a particular solution of the inhomogeneous equation and then to fit initial conditions. This is all done in one step. The dependence of the solution on the initial conditions is explicitly obtained.

We turn to the second-order equation

$$
\left(E^{2}+\alpha_{1} E+\alpha_{2}\right) f=g .
$$

Using

$$
E^{2} f=\frac{1}{\omega^{2}} f-\frac{1}{\omega^{2}} f(0)-\frac{1}{\omega} f(1), \quad E f=\frac{1}{\omega} f-\frac{1}{\omega} f(0),
$$

we find that

$$
f=\frac{f(0)\left(1+\alpha_{1} \omega\right)+f(1) \omega}{1+\alpha_{1} \omega+\alpha_{2} \omega^{2}}+\frac{\omega^{2} g}{1+\alpha_{1} \omega+\alpha_{2} \omega^{2}} .
$$


Let the zeros of the characteristic polynomial $\omega^{2}+\alpha_{1} \omega+\alpha_{2}$ be $\rho_{1}$ and $\rho_{2}$ with $\rho_{1} \neq \rho_{2}$. The partial fraction expansion (see Appendix A) of (7.7) leads to

$$
\begin{aligned}
f(\lambda) & =\frac{1}{\rho_{2}-\rho_{1}}[G(\lambda)+S(\lambda)], \\
G(\lambda) & =f(0)\left[\rho_{2}^{\lambda+1}-\rho_{1}^{\lambda+1}+\alpha_{1}\left(\rho_{2}^{\lambda}-\rho_{1}^{\lambda}\right)\right]+f(1)\left[\rho_{2}^{\lambda}-\rho_{1}^{\lambda}\right] \\
& =f(0)\left[\rho_{2} \rho_{1}^{\lambda}-\rho_{1} \rho_{2}^{\lambda}\right]+f(1)\left[\rho_{2}^{\lambda}-\rho_{1}^{\lambda}\right], \\
S(\lambda) & =\sum_{j=0}^{\lambda-1} g(j)\left(\rho_{2}^{\lambda-1-j}-\rho_{1}^{\lambda-1-j}\right) .
\end{aligned}
$$

Let the characteristic polynomial have a double root at $\rho_{1}$. Ứsing

$$
\frac{1}{\left(1-\rho_{1} \omega\right)^{2}}=(\lambda+1) \rho_{1}^{\lambda}
$$

it is easy to show that

$$
f(\lambda)=\rho_{1}^{\lambda-1}\left\{f(0)\left[\rho_{1}(1-\lambda)\right]+f(1) \lambda\right\}+\sum_{j=0}^{\lambda-2}(\lambda-1-j) \rho_{1}^{\lambda-2-j} g(j) .
$$

The same result may be obtained from (7.8) by calculating the limit as $\rho_{2} \rightarrow \rho_{1}$.

Observe that $\rho_{1}$ and $\rho_{2}$ are, in general, complex. If the coefficients and the starting values are real, the solution is real for all $\lambda$. In the form of the solution given by (7.8) it is not clear that $f$ is real. We give an example below to show that all calculations may be done over the reals.

We turn to the $n$ th-order equation

$$
\sum_{j=0}^{n} \alpha_{n-j} E^{j} f=g, \quad \alpha_{0}=1 .
$$

Using (4.9),

$$
\begin{gathered}
E^{j} f=\tau^{j} f-\sum_{k=0}^{j-1} \tau^{j-k} f(k), \\
\sum_{j=0}^{n} \alpha_{n-j} \tau^{j} f=\sum_{j=0}^{n} \alpha_{n-j} \sum_{k=0}^{j-1} \tau^{j-k} f(k)+g .
\end{gathered}
$$

Interchanging the order of summation and setting $\tau=1 / \omega$, we find

$$
\sum_{j=0}^{n} \alpha_{j} \omega^{j} f=\sum_{k=0}^{n-1} \omega^{k} f(k) \sum_{j=0}^{n-1-k} \alpha_{j} \omega^{j}+\omega^{n} g .
$$

Let

$$
P_{r}(\omega)=\sum_{j=0}^{r} \alpha_{j} \omega^{j}
$$

Then the general solution may be written simply as

$$
f=\frac{\sum_{k=0}^{n-1} \omega^{k} P_{n-1-k}(\omega) f(k)}{P_{n}(\omega)}+\frac{\omega^{n} g}{P_{n}(\omega)} .
$$


Assume that the roots of the characteristic polynomial are distinct. Let

$$
C_{r}(\omega)=\sum_{j=0}^{r} \alpha_{r-j} \omega^{j}
$$

A calculation shows that

Furthermore,

$$
\begin{aligned}
\frac{\omega^{k} P_{n-1-k}(\omega)}{P_{n}(\omega)} & =\sum_{i=1}^{n} \frac{A_{i}}{1-\rho_{i} \omega}, \\
A_{i} & =-\rho_{i}^{1-k} \frac{P_{n-1-k}\left(\frac{1}{\rho_{i}}\right)}{P_{n}^{\prime}\left(\frac{1}{\rho_{i}}\right)}=\frac{C_{n-1-k}\left(\rho_{i}\right)}{C_{n}^{\prime}\left(\rho_{i}\right)} .
\end{aligned}
$$

Therefore,

$$
\begin{aligned}
\frac{\omega^{n-1}}{P_{n}(\omega)} & =\sum_{i=1}^{n} \frac{B_{i}}{1-\rho_{i} \omega}, \\
B_{i} & =-\frac{\rho_{i}{ }^{2-n}}{P_{n}{ }^{\prime}\left(\frac{1}{\rho_{i}}\right)}=\frac{1}{C_{n}{ }^{\prime}\left(\rho_{i}\right)}
\end{aligned}
$$

$$
f(\lambda)=\sum_{k=0}^{n-1} f(k) \sum_{i=1}^{n} \frac{C_{n-1-k}\left(\rho_{i}\right) \rho_{i}{ }^{\lambda}}{C_{n}{ }^{\prime}\left(\rho_{i}\right)}+\sum_{k=0}^{\lambda-1} g(k) \sum_{i=1}^{n} \frac{\rho_{i}^{\lambda-1-k}}{C_{n}{ }^{\prime}\left(\rho_{i}\right)}
$$

or

$$
f(\lambda)=\sum_{i=1}^{n} \rho_{i}^{\lambda} \frac{1}{C_{n}{ }^{\prime}\left(\rho_{i}\right)} \sum_{k=0}^{n-1} C_{n-1-k}\left(\rho_{i}\right) f(k)+\sum_{k=0}^{\lambda-1} g(k) \sum_{i=1}^{n} \frac{\rho_{i}^{\lambda-1-k}}{C_{n}{ }^{\prime}\left(\rho_{i}\right)} .
$$

It follows from (7.11) that

$$
C_{r+1}(\omega)=\omega C_{r}(\omega)+\alpha_{r+1} .
$$

Assume that $\rho_{i}$ is real. For $\rho_{i}$ fixed, all the $C_{n-1-k}\left(\rho_{i}\right)$ may be obtained by synthetic division with about $n$ multiplications. Using the $C_{n-1-k}\left(\rho_{i}\right), C_{n}^{\prime}\left(\rho_{i}\right)$ may also be obtained by synthetic division. (For material on synthetic division, the reader is referred to Kunz [15, pp. 19-21].)

We turn to some examples. The first example shows how all calculations may be done over the reals. Given

$$
\left(E^{2}+2 E+4\right) f=0,
$$

the solution is

$$
f=\frac{f(0)(1+2 \omega)+f(1) \omega}{1+2 \omega+4 \omega^{2}} .
$$

Writing $1+2 \omega=1+\omega+\omega$ and using (3.14) and (3.15), or using (3.18) and (3.19), we obtain

$$
f(\lambda)=2^{\lambda}\left[f(0)\left(\cos \frac{2}{3} \pi \lambda+\frac{1}{\sqrt{ } 3} \sin \frac{2}{3} \pi \lambda\right)+f(1) \frac{1}{\sqrt{ } 3} \sin \frac{2}{3} \pi \lambda\right] .
$$


To illustrate the importance of distinguishing between constant sequences and scalars, we consider

$$
\left(E^{2}-3 E+2\right) f=1, \quad f(0)=0, \quad f(1)=0 .
$$

The right side of this equation is the constant sequence consisting of ones. The solution is

$$
f=\frac{\omega^{2}}{1-3 \omega+2 \omega^{2}} \frac{1}{1-\omega}=\frac{\omega^{2}}{(1-2 \omega)(1-\omega)^{2}} .
$$

Since

$$
\begin{aligned}
\frac{\omega^{2}}{(1-2 \omega)(1-\omega)^{2}} & =\frac{1}{1-2 \omega}-\frac{1}{(1-\omega)^{2}}, \\
f(\lambda) & =2^{\lambda}-\lambda-1 .
\end{aligned}
$$

As our last example, we consider

$$
\left(E^{2}-1\right) f=\lambda !, \quad f(0)=0, \quad f(5)=1 .
$$

Then

$$
f=\frac{f(0)+f(1) \omega}{1-\omega^{2}}+\frac{\omega^{2}}{1-\omega^{2}} \lambda !
$$

and

$$
f(\lambda)=\frac{1}{2} f(1)\left[1-(-1)^{\lambda}\right]+\frac{1}{2} \sum_{j=0}^{\lambda-1}\left[1-(-1)^{\lambda-1-j}\right] j ! .
$$

Since

$$
\begin{aligned}
& f(5)=1=f(1)+7 \\
& f(\lambda)=-3\left[1-(-1)^{\lambda}\right]+\frac{1}{2} \sum_{j=0}^{\lambda-1}\left[1-(-1)^{\lambda-1-j} \mid j !\right.
\end{aligned}
$$

8. Inverse Operators of the Discrete Calculus: $\Delta^{-1}, M^{-1}$. We define $\Delta^{-1} f$ to be the solution of the first-order equation

$$
\Delta \varphi=f .
$$

Clearly, $\Delta^{-1} f$ is determined only up to an arbitrary constant. Despite the symbolism in common usage, $\Delta^{-1}$ does not commute with $\Delta$. It plays only a secondary role in the theory of generalized sequences just as indefinite integration plays only a secondary role in Mikusiński's theory.

From (7.5),

$$
\Delta^{-1} f=\frac{\Delta^{-1} f(0)}{1-\omega}+\frac{\omega}{1-\omega} f=\sigma \Delta^{-1} f(0)+\omega \sigma f,
$$

where $\Delta^{-1} f(0)$ is just an arbitrary constant. Hence

$$
\Delta^{-1} f(\lambda)=(1)^{\lambda} \Delta^{-1} f(0)+\sum_{j=1}^{\lambda-1} f(j)
$$


where $(1)^{\lambda}$ serves to remind us that this constant is added in for each $\lambda$.

We define $M^{-1} f$ as the solution of

$$
M \varphi=f .
$$

Hence

$$
M^{-1} f=\frac{M^{-1} f(0)}{1+\omega}+\frac{2 \omega}{1+\omega} f=\nu M^{-1} f(0)+2 \omega \nu f
$$

or,

$$
M^{-1} f=(-1)^{\lambda} M^{-1} f(0)+2 \sum_{j=0}^{\lambda-1}(-1)^{\lambda-1-j} f(j)
$$

Thus $M^{-1}$ is related to the operator $\nu$. Observe that whereas $M$ and $M^{-1}$ are not inverses, $\nu$ and $\mu$ are inverses. For material on $\Delta^{-1}$ and $M^{-1}$, the reader is referred to Jordan [13, Chapter III].

As an example, we calculate

$$
\begin{aligned}
M^{-1} a^{\lambda} & =\frac{C}{1+\omega}+\frac{2 \omega}{1+\omega} \frac{1}{1-a \omega} \\
& =C^{\prime}(-1)^{\lambda}+\frac{2}{a+1} a^{\lambda} .
\end{aligned}
$$

9. Heaviside and Dirac Sequences. Let

$$
\begin{array}{ll}
\gamma(\lambda, j)=1, & \lambda \geqq j, \\
\gamma(\lambda, j)=0, & \lambda<j .
\end{array}
$$

Let

$$
\begin{array}{ll}
\delta(\lambda, j)=1, & \lambda=j, \\
\delta(\lambda, j)=0, & \lambda \neq j .
\end{array}
$$

The sequence $\gamma(\lambda, j)$ is the sequence analogue of a unit step function. We call it a Heaviside sequence. The sequence $\delta(\lambda, j)$ is the sequence analogue of an impulse or delta function. We call it a Dirac sequence. We shall show that $\rho$ operating on $\gamma(\lambda, j)$ yields $\delta(\lambda, j)$ in analogy to the continuous case where the derivative of the Heaviside function is the Dirac function. $\delta(\lambda, j)$ is often called a Kronecker symbol.

Observe that

$$
\begin{aligned}
& \gamma(\lambda, j)=\frac{\omega^{j}}{1-\omega}, \\
& \delta(\lambda, j)=\omega^{j} .
\end{aligned}
$$

In particular,

$$
\begin{aligned}
& \gamma(\lambda, 0)=\frac{1}{1-\omega}=\sigma, \\
& \delta(\lambda, 0)=1 .
\end{aligned}
$$


Since $\rho=1-\omega$, the identity

$$
(1-\omega) \frac{\omega^{j}}{1-\omega}=\omega^{j}
$$

implies

$$
\rho \gamma(\lambda, j)=\delta(\lambda, j) \text {. }
$$

Therefore

$$
\gamma(\lambda, j)=\sigma \delta(\lambda, j) .
$$

Using (9.4) and (9.7), we observe that

$$
f=\sum_{j=0}^{\infty} f(j) \omega^{j}
$$

implies

$$
\sigma f=\sum_{j=0}^{\infty} f(j) \gamma(\lambda, j) .
$$

Certain algebraic identities in $\omega$ lead to interesting results. From

$$
(1-\omega)^{k+1} \frac{\omega^{j}}{1-\omega}=(1-\omega)^{k} \omega^{j},
$$

we conclude that

$$
\rho^{k+1} \gamma(\lambda, j)=\left\{(-1)^{\lambda} C(k, \lambda)\right\}\{\delta(\lambda, j)\}
$$

From

$$
\begin{aligned}
\frac{1}{(1-\omega)^{k}} \frac{\omega^{j}}{1-\omega} & =\omega^{j-k} \frac{\omega^{k}}{(1-\omega)^{k+1}}, \\
\sigma^{k} \gamma(\lambda, j) & =\omega^{j-k} C(\lambda, k) .
\end{aligned}
$$

Therefore

$$
\tau^{j} \gamma(\lambda, j)=\tau^{k} \rho^{k} C(\lambda, k) .
$$

In a similar manner we can obtain relations between $C(\lambda, k)$ and $C(k, \lambda)$, with $k$ fixed. From

$$
\frac{\omega^{k}}{(1-\omega)^{k+1}}=\frac{\omega^{k}}{(1-\omega)^{2 k+1}}(1-\omega)^{k}
$$

we obtain

$$
C(\lambda, k)=\omega^{k} \sigma^{2 k+1}(-1)^{\lambda} C(k, \lambda) .
$$

Hence $C(\lambda, k)$ is obtained by a $(2 k+1)$-fold summation of $(-1)^{\lambda} C(k, \lambda)$. As an example, let $k=1$. Note that

$$
(-1)^{\lambda} C(1, \lambda)=1,-1,0,0, \cdots .
$$

The sum of this sequence is $1,0,0, \cdots$. The sum of the new sequence is 1,1 , 
$1, \cdots$. Again taking the sum we obtain $1,2,3, \cdots$. Applying $\omega$ to this and using (3.6), we obtain $0,1,2, \cdots$ which is just $C(\lambda, 1)$, which is the left side of $(9.8)$ for $k=1$.

From (9.8),

$$
(-1)^{\lambda} C(k, \lambda)=\tau^{k} \rho^{2 k+1} C(\lambda, k) .
$$

From the identity

$$
(1+\omega)^{k}=(1+\omega)^{2 k+1} \frac{1}{\omega^{k}} \frac{\omega^{k}}{(1+\omega)^{k+1}}
$$

we obtain

$$
C(k, \lambda)=\tau^{k} \mu^{2 k+1}(-1)^{\lambda} C(\lambda, k) .
$$

Recall that $\mu$ is related to the mean operator.

10. Difference Equations with Heaviside and Dirac Sequences. Difference equations whose forcing term depends on Heaviside or Dirac sequences may now be easily handled. For a discussion of such equations using "classical" operator methods, the reader is referred to Tauber and Dean [21].

Consider the first-order difference equation

$$
(E-\beta) f=\gamma(\lambda, j)
$$

Then

$$
f=\frac{f(0)}{1-\beta \omega}+\frac{\omega^{j+1}}{(1-\omega)(1-\beta \omega)}
$$

and

$$
f(\lambda)=f(0) \beta^{\lambda}+\frac{\beta^{\lambda-j}-1}{\beta-1} \cdot \gamma(\lambda, j) .
$$

The dot in the last term of this equation is used to show that this is not a convolution.

Consider the more general equation

$$
(E-\beta) f=g(\lambda) \cdot \gamma(\lambda, j) .
$$

Using (7.6),

$$
f(\lambda)=f(0) \beta^{\lambda}+\sum_{k=0}^{\lambda-1-j} \beta^{k} g(\lambda-1-k) .
$$

The next two examples illustrate the treatment of an equation whose forcing term contains a Dirac sequence. Let

$$
(E-\beta) f=\delta(\lambda, j) .
$$

Then

$$
\begin{aligned}
f & =\frac{f(0)}{1-\beta \omega}+\frac{\omega^{j+1}}{1-\beta \omega}, \\
f(\lambda) & =f(0) \beta^{\lambda}+\beta^{\lambda-1-j} \cdot \gamma(\lambda, j+1) .
\end{aligned}
$$


The solution of

$$
(E-\beta) f=g(\lambda) \cdot \delta(\lambda, j)
$$

is

$$
f(\lambda)=\beta^{\lambda} f(0)+\beta^{\lambda-1-j} g(j) \gamma(\lambda, j+1) .
$$

11. The Scope of Generalized Sequences. Let $f$ and $g$ be sequences and define $h$ by $f=g h$. If $g(0) \neq 0$, then $h$ is a sequence whose members may be recursively calculated by

$$
f(\lambda)=\sum_{j=0}^{\lambda} h(j) g(\lambda-j) .
$$

Let $g \neq 0$. Then there is an element $g(i) \neq 0$. Therefore

$$
g=\sum_{j=i}^{\infty} g(j) \omega^{j}=\omega^{i} \sum_{j=i}^{\infty} g(j) \omega^{j-i} .
$$

Let

$$
e=g(i), g(i+1), g(i+2), \cdots \text {. }
$$

Then $g=\omega^{i} e$ and

$$
h=\frac{f}{g}=\frac{f}{e} \tau^{i} .
$$

Since $e(0) \neq 0, f / e$ is a sequence and

$$
\frac{f}{e}=\sum_{j=0}^{\infty} C(j) \omega^{j},
$$

where $C(j)$ is determined from

$$
f(\lambda)=\sum_{j=0}^{\lambda} C(j) e(\lambda-j) .
$$

Then

$$
\frac{f}{g}=C(0) \omega^{-i}+C(1) \omega^{-i+1}+\cdots
$$

12. Relation to Other Operational Calculi. Operational calculi date back to the time of Leibnitz, Lagrange, and Laplace. A historical survey may be found in Davis [7]. Important contributions were made by Boole [3] and Heaviside. (For an interesting assessment of Heaviside's work, the reader is referred to Cooper [6].) Contemporary texts on operational calculi include those of Kaplan [14] and Churchill [5].

A number of sequence transforms have been studied. These include the Dirichlet transform (Fort [11])

$$
f(t)=\sum_{j=0}^{\infty} a(j) s^{-t j},
$$


or (Zypkin [22])

$$
f(t)=\sum_{j=0}^{\infty} a(j) e^{-t j}
$$

the $z$-transform (Kaplan [14, pp. 375-388], Lawden [16])

$$
f(z)=\sum_{j=0}^{\infty} a(j) z^{-j}
$$

and the generatrix transform (Riordan [20, Chapter 2])

$$
f(t)=\sum_{j=0}^{\infty} a(j) t^{j}
$$

It is clear that these transforms are closely related. The solution of linear difference equations with constant coefficients has been frequently performed by operational means. The reader is referred to Boole [3, Chapter XI], Jordan [13, Chapter XI], Milne-Thomson [18, Chapter XIII], Mikusitski [17, pp. 158-165] and Erdélyi [9, pp. 70-74].

The idea of multiplication defined by convolution is not new. The reader is referred to an important paper by Bell [1]. The key to Mikusinski's work is the introduction of an inverse to convolution. Moore [19] defines a convolution algebra in order to find formal power series solutions to differential equations. Feldman [10] and Eliáš [8] solve difference equations. Eliáš defines $h=f g$ by

$$
h(\lambda)=\sum_{j=1}^{\lambda} f(\lambda-j) g(j-1), \quad \lambda>0, \quad h(0)=0 .
$$

Berg $[2$, p. 26] defines $h=f g$ by

$$
h(\lambda)=\sum_{j=0}^{\lambda} f(j) g(\lambda-j)-\sum_{j=0}^{-1} f(j) g(\lambda-1-j) .
$$

In a paper published in 1950, Frankel [12] discusses a "calculus of figurate numbers." Frankel does not seem aware of the fact that his calculus is based on the sequences $C(\lambda, j)$ and $(-1)^{\lambda} C(j, \lambda)$.

Thus a number of authors have written on convolution algebras with applications to specific problems. The systematic application of such an algebra to a wide variety of problems in the discrete calculus is the contribution of this paper.

We now investigate the relation between generalized sequences and generating functions. From (3.4), any sequence may be written as

$$
f=\sum_{j=0}^{\infty} f(j) \omega^{j} .
$$

To the sequence with elements $f(j)$ we may assign the generating function

$$
f(t)=\sum_{j=0}^{\infty} f(j) t^{j} .
$$

Note that (12.1) is an identity which follows from the definition of the sequence $\omega$. It is simply a way of exhibiting the sequence in terms of its members and is always true. There can be no question of convergence. Equation (12.2) defines a 
mapping from a sequence to a function of a real variable. There is an obvious isomorphism between the two systems.

The conceptual difference between generating functions and gereralized sequences may be illustrated by the following example. The generating function of the sequence $1, a, a^{2}, \cdots$ is $1 /(1-a t)$ because

$$
\sum_{j=0}^{\infty}(a t)^{j}=\frac{1}{1-a t} .
$$

On the other hand,

$$
\frac{1}{1-a \omega}=1, a, a^{2}, \cdots
$$

by the definition of fraetion in our convolution algebra and by the definition of $\omega$.

In the calculus of generalized sequences the formula for the coefficients of a product polynomial in terms of the coefficients of its factors is built into the system. In a sense, generalized sequences are to generating functions as the method of detached coefficients (synthetic division) is to polynomial division. On the other hand, the relations discovered during almost two hundred years of experience with generating functions may be used in the theory of generalized sequences because of the isomorphism.

Appendix A. On Partial Fraction Expansions. The expansion of rational functions into partial fractions is too well known to require discussion here. The reader may refer to Kaplan [14, pp. 158-160]. The object of this appendix is to point out a simplification in the calculation of the coefficients.

Let $P(x) / Q(x)$ be a proper rational function, and let $Q(x)$ have a zero $\alpha$ of degree $p$. Let

$$
\varphi(x)=(x-\alpha)^{p} \frac{P(x)}{Q(x)} .
$$

Then

where

$$
\frac{P(x)}{Q(x)}=\frac{B_{1}}{x-\alpha}+\frac{B_{2}}{(x-\alpha)^{2}}+\cdots+\frac{B_{p}}{(x-\alpha)^{p}}+\lambda(x)
$$

$$
B_{p-j}=\frac{\varphi^{(j)}(\alpha)}{j !}, \quad j=0,1, \cdots, p-1,
$$

and where $\lambda(x)$ is a partial fraction expansion determined by the other zeros of $Q(x)$. From (A.1) and (A.2), it appears that the coefficients in the expansion of $P(x) / Q(x)$ require the differentiation of a quotient which depends on $P(x)$ and $Q(x)$. We shall demonstrate that if the coefficients in the expansion of $1 / Q(x)$ are $A_{j}$, then the $B_{j}$ are linear combinations of the $A_{j}$ with coefficients depending on $P(x)$ only.

The proof is simple. Let

$$
\frac{1}{Q(x)}=\frac{A_{1}}{x-\alpha}+\frac{A_{2}}{(x-\alpha)^{2}}+\cdots+\frac{A_{p}}{(x-\alpha)^{p}}+\tau,
$$


where

$$
A_{p-j}=\frac{\nu^{(j)}(\alpha)}{j !}, \quad \nu(x)=\frac{(x-\alpha)^{p}}{Q(x)}
$$

Since

$$
\begin{aligned}
\varphi(x) & =P(x) \nu(x), \\
\varphi^{(j)}(x) & =\sum_{k=0}^{j} C(j, k) P^{(j-k)}(x) \nu^{(k)}(x),
\end{aligned}
$$

and

$$
B_{p-j}=\sum_{k=0}^{j} A_{p-k} \frac{P^{(j-k)}(\alpha)}{(j-k) !}, \quad j=0 ; 1, \cdots, p-1 .
$$

Equation (A.3) is the required relation.

Partial fraction expansions occur in the solution of difference equations. In this case, $Q(x)$ is determined only by the coefficients of the homogeneous equation, and $P(x)$ is determined only by the initial conditions and the forcing term. Because of (A.3), the expansion due to the homogeneous equation may be determined and the initial conditions and the forcing term may then be varied without requiring the recalculation of the expansion coefficients from scratch.

Appendix B. Automation of Operator Calculus. The solution of a problem with the aid of an operational calculus bears a resemblance to the process of solving a problem on a computer. First there is a translation phase. For a problem where an operational calculus is used this is typically a translation from an analytic to an algebraic problem. For a computer problem, this is the translation of, say, a mathematical formulation in the language of FORTRAN into machine language. Then the problem is solved. Finally, an inverse translation is needed to make the results available. For the operational calculus, this involves the application of inverse transforms. For a computer problem this might mean binary-to-decimal conversion or conversion to CRT output.

One advantage of an operational calculus lies in the fact that results may be obtained in a mechanical or "cookbook" fashion. This suggests that certain classes of problems could conveniently be solved on computers with an operational calculus providing a natural symbolism. Tables of transforms and inverse transforms would perform the functions of "syntax tables" in compilers. To change from, say, a calculus for difference equations to one for differential equations, it is only necessary to change these tables.

The computer can also function as a repository of knowledge. For this purpose an operational calculus plays a natural role since the contents of certain parts of mathematics may be summarized in the algebraic structure of appropriate operators and in the transform tables.

Some work on symbolic manipulation on digital computers has already been done. A noteworthy example is Brown's [4] ALPAK program which performs symbolic manipulation on rational functions of several variables. The symbolic solution 
of various types of linear systems seems a particularly suitable candidate for automation.

Bell Telephone Laboratories, Incorporated

Murray Hill, New Jersey

1. E. T. Bell, "Euler algebra," Trans. Amer. Math. Soc., v. 25, 1923, pp. 135-154.

2. L. BERG, Einführung in die Operatorenrechnung, Mathematik für Naturwissenschaft und Technik, Bd. 6, VEB Deutscher Verlag der Wissenschaften, Berlin, 1962. MR $25 * 5347$.

3. G. Boole, Calculus of Finite Differences, 4th ed., Chelsea, New York, 1957. MR 20 * 1124 .

4. W. S. Brown, J. P. Hyde, \& B. A. Tague, "The ALPAK system for nonnumerical algebra on a digital computer. II. Rational functions on several variables," Bell System Tech. $J .$, v. 43,1964 , pp. $785-804$.

5. R. V. ChURChILl, Operational Mathematics, McGraw-Hill, New York, 1958. MR 21 *7411.

6. J. L. B. CoOPER, "Heaviside and the operational calculus," Math. Gaz., v. 36, 1952, pp. 5-19. MR 13, 612 . 1936.

7. H. T. DAvis, The Theory of Linear Operators, Principia Press, Bloomington, Indiana,

8. J. ELIÁš, "On an operator method for difference equations" (Czech), Mat.-Fys. Casopis. Sloven. Akad. Vied., v. 8, 1958, pp. 203-227. MR 21 *4312.

9. A. ERdElyi, Operational Calculus and Generalized Functions, Holt, Rinehart and Winston, New York, 1962.

10. L. Feldman, "On linear difference equations with constant coefficients," Periodica Polytech. Elec. Engrg., v. 3, 1959, pp. 247-257.

11. T. ForT, "Linear difference equations and the Dirichlet transform," Amer. Math. Monthly, v. 62, 1955, pp. 641-645.

12. E. T. FrankeL, "A calculus of figurate numbers and finite differences," Amer. Math. Monthly, v. 57, 1950, pp. 14-25. MR 11, 412 .

13. C. Jordan, Calculus of Finite Differences, Chelsea, New York, 1950.

14. W. Kaplan, Operational Methods for Linear Systems, Addison-Wesley Series in Mathematics, Addison-Wesley, Reading, Mass., 1962. MR $25 * 3330$.

15. K. S. KUnz, Numerical Analysis, McGraw-Hill, New York, 1957. MR 19, 460.

16. D. F. LAwDEN, "On the solution of linear difference equations," Math. Gaz., v. 36, 1952, pp. 193-196. MR 14, 285.

17. J. Mikusi אski, Operational Calculus, International Series of Monographs on Pure and Applied Mathematics, Vol. 8, Pergamon, New York; Panstwowe Wydawnictwo, Naukowe, Warsaw, 1959. MR 21 * 4333.

18. L. M. Minne-Thomson, The Calculus of Finite Differences, Macmillan, London, 1933.

19. D. H. Moore, "Convolution products and quotients and algebraic derivatives of sequences," Amer. Math. Monthly, v. 69, 1962, pp. 132-138. MR $25 * 402$.

20. J. Riordan, An Introduction to Combinatorial Analysis, Wiley Publications in Mathematical Statistics, Wiley, New York and Chapman \& Hall, London, 1958. MR $20 * 3077$.

21. S. TAUBER \& D. DEAN, "On difference equations containing step and delta functions," J. Soc. Indust. Appl. Math., v. 8, 1960, pp. 174-180. MR $22 * 5832$.

22. J. S. Z Y PKIN, Differenzenrechnungen der Impulse und Regeltechnik (Translated from Russian), Verlag Technik, Berlin, 1956. 